\title{
Christoffel Transforms and Hermitian Linear Functionals
}

\author{
Francisco Marcellán and Javier Hernández
}

\begin{abstract}
In this contribution we are focused on some spectral transformations of Hermitian linear functionals. They are the analogues of the Christoffel transform for linear functionals, i. e. for Jacobi matrices which has been deeply studied in the past. We consider Hermitian linear functionals associated with a probability measure supported on the unit circle. In such a case we compare the Hessenberg matrices associated with such a probability measure and its Christoffel transform. In this way, almost unitary matrices appear. We obtain the deviation to the unit matrix both for principal submatrices and the complete matrices respectively.
\end{abstract}

Mathematics Subject Classification (2000). Primary 42C05; Secondary 15A23. Keywords. Hermitian linear functionals, Christoffel transforms, Laurent polynomials, orthogonal polynomials, QR factorization.

\section{Introduction}

\subsection{Christoffel transforms on the unit circle}

Let $u$ be a linear functional in the linear space $\Lambda$ of Laurent polynomials with complex coefficients, i. e. $\Lambda=\operatorname{span}\left\{z^{n}\right\}_{n \in \mathbb{Z}} \cdot u$ is said to be Hermitian if

$$
\left\langle u, z^{-n}\right\rangle=\overline{\left\langle u, z^{n}\right\rangle} \text { for every } n \in \mathbb{N} .
$$

Let $S$ be the Hermitian bilinear form in $\mathrm{P}$ such that

$$
\mathcal{S}(p, q)=\left\langle u, p(z) \bar{q}\left(z^{-1}\right)\right\rangle, \quad p, q \in \mathbb{P} .
$$

Let $T$ denote the Gram matrix of $S$ with respect to the canonical basis $\left\{z^{n}\right\}_{n \in \mathbb{N}}$. The entries $t_{m, n}$ of $T$ are

$$
t_{m, n}=\left\langle u, z^{m-n}\right\rangle
$$

In other words, $T$ is an Hermitian Toeplitz matrix. $T_{n}$ will denote the leading principal submatrix of $T$ of order $n$. We will assume $t_{0,0}=1$. 
Definition $1.1([2])$.

(i) $\mathcal{S}$ is said to be quasi-definite if $T_{n}$ is nonsingular for every $n \in \mathbb{N}$.

(ii) $\mathcal{S}$ is said to be positive definite if $\operatorname{det} T_{n}>0$ for every $n \in \mathbb{N}$.

In a positive definite case it is very well known (see [2], [6]) that $u$ has an integral representation

$$
\langle u, p(z)\rangle=\int_{0}^{2 \pi} p\left(\mathrm{e}^{i \theta}\right) \mathrm{d} \mu(\theta),
$$

where $\mu$ is a nontrivial probability measure.

Proposition 1.2. $\mathcal{S}$ is quasi-definite (resp. positive definite) if and only if there exists a sequence of monic polynomials $\left\{P_{n}\right\}_{n \in \mathbb{N}}$ with

(i) $\operatorname{deg} P_{n}=n$,

(ii) $S\left(P_{n}, P_{m}\right)=\mathbf{k}_{n} \delta_{n, m}$ with $\mathbf{k}_{n} \neq 0$ (resp. $\mathbf{k}_{n}>0$ ).

The sequence $\left\{P_{n}\right\}_{n \in \mathbb{N}}$ is said to be the sequence of monic orthogonal polynomials with respect to $\mathcal{S}$. $\left\{P_{n}\right\}_{n \in \mathbb{N}}$ satisfies the Szegö recurrence relations ( see $[2],[6])$

$$
\begin{gathered}
P_{n+1}(z)=z P_{n}(z)+P_{n+1}(0) P_{n}^{*}(z), n \geqslant 0, \\
P_{n+1}(z)=\left(1-\left|P_{n+1}(0)\right|^{2}\right) z P_{n}(z)+P_{n+1}(0) P_{n+1}^{*}(z), n \geqslant 0,
\end{gathered}
$$

where $P_{n}^{*}(z)=z^{n} \bar{P}_{n}\left(z^{-1}\right)$ is said to be the reversed polynomial of $P_{n}$ (see [2], [6]). The $n$-th kernel $K_{n}(z, y)$ associated with $\mathcal{S}$ is defined by

$$
K_{n}(z, y)=\sum_{j=0}^{n} \frac{P_{j}(z) \overline{P_{j}(y)}}{\mathbf{k}_{j}} .
$$

In the positive definite case, the $n$-th kernel polynomial is associated with the following extremal problem

$$
\min \left\{\int_{0}^{2 \pi}\left|p\left(\mathrm{e}^{i \theta}\right)\right|^{2} \mathrm{~d} \mu(\theta): \operatorname{deg} p \leqslant n, p(y)=1\right\} .
$$

Indeed, the value of this minimum is

$$
\lambda_{n}(y)=\frac{1}{K_{n}(y, y)} .
$$

For each complex number $y, \lambda_{n}$ is decreasing in $n$ and thus we can define

$$
\begin{aligned}
\lambda_{\infty}(y) & =\lim _{n \rightarrow \infty} \lambda_{n}(y) \\
& =\inf \left\{\int_{0}^{2 \pi}\left|p\left(\mathrm{e}^{i \theta}\right)\right|^{2} \mathrm{~d} \mu(\theta): p \in \mathbb{P}, p(y)=1\right\} \geqslant 0 .
\end{aligned}
$$

$\lambda_{\infty}$ is said to be the Christoffel function associated with $\mu$. One of the main results about the behavior of the Christoffel function is the following. 
Proposition 1.3 ([9], Thm. 2.2.1). Let $\mu$ be a nontrivial probability measure on the unit circle. Then

(i) If $|y|>1$, then $\lambda_{\infty}(y)=0$.

(ii) If $|y|=1$, then $\lambda_{\infty}(y)=\mu(\{y\})$.

(iii) If $\sum_{n=0}^{\infty}\left|P_{n}(0)\right|^{2}=+\infty$, then $\lambda_{\infty}(y)=0$ for every $y$ with $|y|<1$.

(iv) If $\sum_{n=0}^{\infty}\left|P_{n}(0)\right|^{2}<+\infty$, then $\lambda_{\infty}(y)>0$ for every $y$ with $|y|<1$. Furthermore,

$$
\lambda_{\infty}(0)=\prod_{n=1}^{\infty}\left(1-\left|P_{n}(0)\right|^{2}\right) .
$$

If $P=\left[P_{0}(z), P_{1}(z), \ldots\right]^{t}$, then there exists a lower Hessenberg matrix $H_{P}$ such that

$$
z P=H_{P} P
$$

The entries $h_{l, j}$ of $H_{P}$ are

$$
h_{l, j}=\left\{\begin{array}{lll}
-\frac{\mathbf{k}_{l}}{\mathbf{k}_{j}} P_{l+1}(0) \overline{P_{j}(0)}, & \text { if } & 0 \leqslant j \leqslant l \\
1, & \text { if } & j=l+1 \\
0, & & \text { otherwise }
\end{array}\right.
$$

Next, consider the Hermitian bilinear form

$$
\mathcal{S}_{2}(p, q):=\mathcal{S}((z-\alpha) p,(z-\alpha) q), \quad p, q \in \mathbb{P} .
$$

\section{Proposition 1.4 ([8]).}

(i) $\mathcal{S}_{2}$ is quasi-definite if and only if $K_{n}(\alpha, \alpha) \neq 0$ for every $n \in \mathbb{N}$.

(ii) If $\left\{Q_{n}(z)\right\}_{n \in \mathbb{N}}$ denotes the sequence of monic orthogonal polynomials with respect to $\mathcal{S}_{2}$, then

$$
Q_{n}(z)=\frac{1}{z-\alpha}\left(P_{n+1}(z)-\frac{P_{n+1}(\alpha)}{K_{n}(\alpha, \alpha)} K_{n}(z, \alpha)\right) .
$$

$\mathcal{S}_{2}$ is said to be the canonical Christoffel transform of the bilinear form $\mathcal{S}$.

\section{Polynomial perturbations of positive measures}

If $\mathcal{S}$ is a positive definite bilinear form, then $\mathcal{S}_{2}$ is also a positive definite bilinear form [3]. We can introduce the sequence of orthonormal polynomials $\left\{\varphi_{n}\right\}_{n \in \mathbb{N}}$ associated with $\mathcal{S}$, where

$$
\varphi_{n}(z)=\mathbf{k}_{n}^{-\frac{1}{2}} P_{n}(z)
$$

We will denote $\left\{\psi_{n}\right\}_{n \in \mathbb{N}}$ the corresponding sequence of orthonormal polynomials associated with $\mathcal{S}_{2}$. Thus we get

$$
(z-\alpha) \psi(z)=M \varphi(z)
$$


where $\psi(z)=\left[\psi_{0}(z), \psi_{1}(z), \ldots\right]^{t}, \varphi(z)=\left[\varphi_{0}(z), \varphi_{1}(z), \ldots\right]^{t}$, and $M$ is a lower Hessenberg matrix with entries $m_{l, j}$ given by

$$
m_{l, j}= \begin{cases}-\frac{\varphi_{l+1}(\alpha) \overline{\varphi_{j}(\alpha)}}{\sqrt{K_{l+1}(\alpha, \alpha) K_{l}(\alpha, \alpha)}}, & \text { if } j \leqslant l, \\ \sqrt{\frac{K_{l}(\alpha, \alpha)}{K_{l+1}(\alpha, \alpha)}}, & \text { if } j=l+1, \\ 0, & \text { if } j>l+1 .\end{cases}
$$

\section{Proposition 2.1.}

(i) $M M^{*}=I$.

(ii) $M^{*} M=I-\lambda_{\infty}(\alpha) \varphi(\alpha) \varphi^{*}(\alpha)$.

where $I$ is the infinite unit matrix.

Proof. (i) From the orthogonality of $\left\{\varphi_{n}\right\}_{n \in \mathbb{N}}$ and $\left\{\psi_{n}\right\}_{n \in \mathbb{N}}$ with respect to $\mathcal{S}$ and $\mathcal{S}_{2}$ respectively, we get

$$
\begin{aligned}
I & =\mathcal{S}_{2}\left(\psi(z), \psi^{t}(z)\right)=\mathcal{S}\left((z-\alpha) \psi(z),(z-\alpha) \psi^{t}(z)\right) \\
& =\mathcal{S}\left(M \varphi(z), \varphi^{t}(z) M^{t}\right)=M \mathcal{S}\left(\varphi(z), \varphi^{t}(z)\right) M^{*}=M M^{*} .
\end{aligned}
$$

(ii) For $j=0,1, \ldots$

$$
\begin{aligned}
M_{(j)}^{*} M^{(j)} & =\frac{K_{j-1}(\alpha, \alpha)}{K_{j}(\alpha, \alpha)}+\left|\varphi_{j}(\alpha)\right|^{2} \sum_{l=j}^{\infty} \frac{\left|\varphi_{l+1}(\alpha)\right|^{2}}{K_{l+1}(\alpha, \alpha) K_{l}(\alpha, \alpha)} \\
& =\frac{K_{j-1}(\alpha, \alpha)}{K_{j}(\alpha, \alpha)}+\left|\varphi_{j}(\alpha)\right|^{2} \sum_{l=j}^{\infty}\left(\frac{1}{K_{l}(\alpha, \alpha)}-\frac{1}{K_{l+1}(\alpha, \alpha)}\right) \\
& =1-\lambda_{\infty}(\alpha)\left|\varphi_{j}(\alpha)\right|^{2} .
\end{aligned}
$$

For $k<j$

$$
\begin{aligned}
M_{(k)}^{*} M^{(j)} & =-\frac{1}{K_{j}(\alpha, \alpha)} \varphi_{k}(\alpha) \overline{\varphi_{j}(\alpha)}+\varphi_{k}(\alpha) \overline{\varphi_{j}(\alpha)} \sum_{l=j}^{\infty} \frac{\left|\varphi_{l+1}(\alpha)\right|^{2}}{K_{l+1}(\alpha, \alpha) K_{l}(\alpha, \alpha)} \\
& =\varphi_{k}(\alpha) \overline{\varphi_{j}(\alpha)}\left(-\frac{1}{K_{j}(\alpha, \alpha)}+\sum_{l=j}^{\infty}\left(\frac{1}{K_{l}(\alpha, \alpha)}-\frac{1}{K_{l+1}(\alpha, \alpha)}\right)\right) \\
& =-\varphi_{k}(\alpha) \overline{\varphi_{j}(\alpha)} \lambda_{\infty}(\alpha) .
\end{aligned}
$$

According to Proposition 1.3, $M$ is a unitary matrix if $|y|>1$, and for $|y|=1$, $\lambda_{\infty}(y)=0$. Furthermore, if $|y|<1$ and $\sum_{n=0}^{\infty}\left|P_{n}(0)\right|^{2}=+\infty$, then $M$ is unitary.

An analogous result for the leading principal submatrices of $M$ is the following.

Proposition 2.2. Let $M_{n}$ be the leading principal submatrix of order $n$ of $M$, then

(i) $M_{n} M_{n}^{*}=I_{n}-\frac{K_{n-1}(\alpha, \alpha)}{K_{n}(\alpha, \alpha)} e_{n} e_{n}^{*}$, where $I_{n}$ denotes the unit matrix of order $n$ and $e_{n}=[0, \ldots, 0,1]^{t}$ is a column vector of order $n$.

(ii) $M_{n}^{*} M_{n}=I_{n}-\frac{1}{K_{n}(\alpha, \alpha)} \Phi_{n} \Phi_{n}^{*}$, where $\Phi_{n}=\left[\varphi_{0}(\alpha), \varphi_{1}(\alpha), \ldots, \varphi_{n-1}(\alpha)\right]^{t}$. 
The matrix $M_{n}$ is said to be "almost" unitary (see [7], [9]) in the sense that its first $n-1$ rows constitute an orthonormal set and the last row is orthogonal to this set, but is not normalized.

Proof. (i) For $0 \leqslant k \leqslant n-2$, we deduce

$$
\begin{aligned}
\left(M_{n}\right)_{(k)}\left(M_{n}^{*}\right)^{(k)} & =\frac{\left|\varphi_{k+1}(\alpha)\right|^{2}}{K_{k+1}(\alpha, \alpha) K_{k}(\alpha, \alpha)} \sum_{l=0}^{k}\left|\varphi_{l}(\alpha)\right|^{2}+\frac{K_{k}(\alpha, \alpha)}{K_{k+1}(\alpha, \alpha)} \\
& =\frac{\left|\varphi_{k+1}(\alpha)\right|^{2}}{K_{k+1}(\alpha, \alpha) K_{k}(\alpha, \alpha)} K_{k}(\alpha, \alpha)+\frac{K_{k}(\alpha, \alpha)}{K_{k+1}(\alpha, \alpha)}=1 .
\end{aligned}
$$

On the other hand,

$$
\begin{aligned}
\left(M_{n}\right)_{(n-1)}\left(M_{n}^{*}\right)^{(n-1)} & =\frac{\left|\varphi_{n}(\alpha)\right|^{2}}{K_{n}(\alpha, \alpha) K_{n-1}(\alpha, \alpha)} \sum_{l=0}^{n-1}\left|\varphi_{l}(\alpha)\right|^{2} \\
& =\frac{\left|\varphi_{n}(\alpha)\right|^{2}}{K_{n}(\alpha, \alpha)}=1-\frac{K_{n-1}(\alpha, \alpha)}{K_{n}(\alpha, \alpha)} .
\end{aligned}
$$

Now, for $k<j$, we get

$$
\begin{aligned}
& \left(M_{n}\right)_{(k)}\left(M_{n}^{*}\right)^{(j)} \\
& =\sum_{l=0}^{k} \frac{\varphi_{k+1}(\alpha) \overline{\varphi_{j+1}(\alpha)}\left|\varphi_{l}(\alpha)\right|^{2}}{\sqrt{K_{k+1}(\alpha, \alpha) K_{k}(\alpha, \alpha)} \sqrt{K_{j+1}(\alpha, \alpha) K_{j}(\alpha, \alpha)}}-\frac{\varphi_{k+1}(\alpha) \overline{\varphi_{j+1}(\alpha)}}{\sqrt{K_{j+1}(\alpha, \alpha) K_{j}(\alpha, \alpha)}} \sqrt{\frac{K_{k}(\alpha, \alpha)}{K_{k+1}(\alpha, \alpha)}} \\
& =\frac{\varphi_{k+1}(\alpha) \overline{\varphi_{j+1}(\alpha)}}{\sqrt{K_{k+1}(\alpha, \alpha) K_{k}(\alpha, \alpha) K_{j+1}(\alpha, \alpha) K_{j}(\alpha, \alpha)}}\left(\sum_{l=0}^{k}\left|\varphi_{l}(\alpha)\right|^{2}-K_{i}(\alpha, \alpha)\right)=0 .
\end{aligned}
$$

(ii) For $1 \leqslant k \leqslant n-1$,

$$
\begin{aligned}
\left(M_{n}^{*}\right)_{(k)}\left(M_{n}\right)^{(k)} & =\frac{K_{k-1}(\alpha, \alpha)}{K_{k}(\alpha, \alpha)}+\sum_{l=k}^{n-1} \frac{\left|\varphi_{k}(\alpha)\right|^{2}\left|\varphi_{l+1}(\alpha)\right|^{2}}{K_{l+1}(\alpha, \alpha) K_{l}(\alpha, \alpha)} \\
& =\frac{K_{k-1}(\alpha, \alpha)}{K_{k}(\alpha, \alpha)}+\left|\varphi_{k}(\alpha)\right|^{2} \sum_{l=k}^{n-1}\left(\frac{1}{K_{l}(\alpha, \alpha)}-\frac{1}{K_{l+1}(\alpha, \alpha)}\right) \\
& =\frac{K_{k-1}(\alpha, \alpha)}{K_{k}(\alpha, \alpha)}+\left|\varphi_{k}(\alpha)\right|^{2}\left(\frac{1}{K_{k}(\alpha, \alpha)}-\frac{1}{K_{n}(\alpha, \alpha)}\right) \\
& =1-\frac{\left|\varphi_{k}(\alpha)\right|^{2}}{K_{n}(\alpha, \alpha)},
\end{aligned}
$$

as well as

$$
\begin{aligned}
\left(M_{n}^{*}\right)_{(0)}\left(M_{n}\right)^{(0)} & =\sum_{l=0}^{n-1} \frac{\left|\varphi_{0}(\alpha)\right|^{2}\left|\varphi_{l+1}(\alpha)\right|^{2}}{K_{l+1}(\alpha, \alpha) K_{l}(\alpha, \alpha)} \\
& =\left|\varphi_{0}(\alpha)\right|^{2} \sum_{l=0}^{n-1}\left(\frac{1}{K_{l}(\alpha, \alpha)}-\frac{1}{K_{l+1}(\alpha, \alpha)}\right) \\
& =1-\frac{1}{K_{n}(\alpha, \alpha)} .
\end{aligned}
$$


Now, for $k<j$,

$$
\begin{aligned}
\left(M_{n}^{*}\right)_{(k)}\left(M_{n}\right)^{(j)} & =-\frac{\varphi_{k}(\alpha) \overline{\varphi_{j}(\alpha)}}{\sqrt{K_{j}(\alpha, \alpha) K_{j-1}(\alpha, \alpha)}} \sqrt{\frac{K_{j-1}(\alpha, \alpha)}{K_{j}(\alpha, \alpha)}}+\sum_{l=k}^{n-1} \frac{\left|\varphi_{l+1}(\alpha)\right|^{2} \varphi_{k}(\alpha) \overline{\varphi_{j}(\alpha)}}{K_{l+1}(\alpha, \alpha) K_{l}(\alpha, \alpha)} \\
& =-\frac{\varphi_{k}(\alpha) \overline{\varphi_{j}(\alpha)}}{K_{j}(\alpha, \alpha)}+\varphi_{k}(\alpha) \overline{\varphi_{j}(\alpha)} \sum_{l=j}^{n-1} \frac{\left|\varphi_{l+1}(\alpha)\right|^{2}}{K_{l+1}(\alpha, \alpha) K_{l}(\alpha, \alpha)} \\
& =-\varphi_{k}(\alpha) \overline{\varphi_{j}(\alpha)}\left(\frac{1}{K_{j}(\alpha, \alpha)}-\sum_{l=j}^{n-1}\left(\frac{1}{K_{l}(\alpha, \alpha)}-\frac{1}{K_{l+1}(\alpha, \alpha)}\right)\right) \\
& =-\frac{\varphi_{k}(\alpha) \overline{\varphi_{j}(\alpha)}}{K_{n}(\alpha, \alpha)} .
\end{aligned}
$$

Notice that for $\alpha=0, \varphi_{n}=\psi_{n}$ for every $n \in \mathbb{N}$. Thus $M_{n}=\left(H_{\varphi}\right)_{n}$ and we recover some very well known results of the literature (see [5], [7], [9]).

Let $L$ be the lower triangular matrix such that $\varphi(z)=L \psi(z)$.

\section{Proposition 2.3 ([8]).}

(i) $L=\left(H_{\varphi}-\alpha I\right) M^{*}$.

(ii) $H_{\psi}-\alpha I=M L$.

Therefore, to compute $H_{\psi}-\alpha I$, we consider the QR-factorization of the matrix $\left(H_{\varphi}-\alpha I\right)^{*}$, i. e. we assume the column vectors are linearly independent.

$$
\left(H_{\varphi}-\alpha I\right)^{*}=\tilde{Q} \tilde{R}
$$

where $\tilde{Q}$ has orthonormal column vectors and $\tilde{R}$ is an upper triangular matrix with nonnegative diagonal entries. Hence

$$
H_{\varphi}-\alpha I=\tilde{R}^{*} \tilde{Q}^{*} .
$$

Proposition $2.4([8]) . L=\tilde{R}^{*}$ and $M=\tilde{Q}^{*}$.

This is the unit circle analog of the result obtained in [1] for Jacobi matrices.

Example. We consider the bilinear forms

$$
\begin{aligned}
\mathcal{S}(p, q) & =\int_{0}^{2 \pi} p\left(\mathrm{e}^{i \theta}\right) \overline{q\left(\mathrm{e}^{i \theta}\right)} \frac{1}{\left|\mathrm{e}^{i \theta}-\beta\right|^{2}} \frac{\mathrm{d} \theta}{2 \pi} \text { and } \\
\mathcal{S}_{2}(p, q) & =\int_{0}^{2 \pi} p\left(\mathrm{e}^{i \theta}\right) \overline{q\left(\mathrm{e}^{i \theta}\right)} \frac{\left|\mathrm{e}^{i \theta}-\alpha\right|^{2}}{\left|\mathrm{e}^{i \theta}-\beta\right|^{2}} \frac{\mathrm{d} \theta}{2 \pi},
\end{aligned}
$$

with $|\beta|<1$ and $|\alpha|=1$ (see [4]). It is straightforward to prove that the sequence $\left\{\varphi_{n}\right\}_{n \in \mathbb{N}}$ is given by

$$
\varphi_{0}(z)=\left(1-|\beta|^{2}\right)^{\frac{1}{2}}, \quad \varphi_{n}(z)=z^{n-1}(z-\beta), \text { for } n \geqslant 1 .
$$


Hence

$$
H_{\varphi}-\alpha I=\left[\begin{array}{ccccc}
\beta-\alpha & \left(1-|\beta|^{2}\right)^{\frac{1}{2}} & 0 & \cdots & \\
0 & -\alpha & 1 & 0 & \\
0 & 0 & -\alpha & 1 & \ddots \\
\vdots & & \ddots & \ddots & \ddots
\end{array}\right],
$$

and the $n$-th kernel polynomial $K_{n}(z, \alpha)$ associated with $\mathcal{S}$ is

$$
K_{n}(z, \alpha)=1-|\beta|^{2}+\sum_{j=1}^{n}(z-\beta) \overline{(\alpha-\beta)}\left(\frac{z}{\alpha}\right)^{j-1} .
$$

As a consequence

$$
K_{n}(\alpha, \alpha)=1-|\beta|^{2}+n|\alpha-\beta|^{2} .
$$

The entries $m_{k, j}$ of $M$ are

$$
m_{l, j}=\left\{\begin{array}{llc}
-\frac{\alpha^{l}(\alpha-\beta)\left(1-|\beta|^{2}\right)^{\frac{1}{2}}}{\sqrt{K_{l}(\alpha, \alpha) K_{l+1}(\alpha, \alpha)}}, & \text { if } & j=0, \\
-\frac{\alpha-\left.\beta\right|^{2} \alpha^{l-j+1}}{\sqrt{K_{l}(\alpha, \alpha) K_{l+1}(\alpha, \alpha)}}, & \text { if } & 1 \leqslant j \leqslant l, \\
\sqrt{\frac{K_{l}(\alpha, \alpha)}{K_{l+1}(\alpha, \alpha)}}, & \text { if } & j=l+1, \\
0, & & \text { otherwise. }
\end{array}\right.
$$

From (i) of Proposition 2.3, the matrix $L$ is obtained by means of the expression $L=\left(H_{\varphi}-\alpha I\right) M^{*}$. From this we obtain a lower bidiagonal matrix with entries

$$
l_{r, j}= \begin{cases}\sqrt{K_{1}(\alpha, \alpha)}, & j=r=0, \\ -\alpha \sqrt{\frac{K_{r-1}(\alpha, \alpha)}{K_{r}(\alpha, \alpha)}}, & j=r-1, \\ \sqrt{\frac{K_{r+1}(\alpha, \alpha)}{K_{r}(\alpha, \alpha)}}, & j=r, \\ 0, & \text { otherwise. }\end{cases}
$$

Thus, by (ii) of Proposition 2.3, the entries $\tilde{h}_{r, j}$ of the matrix $H_{\psi}-\alpha I$ are

$$
\tilde{h}_{r, j}= \begin{cases}-\left(\alpha-\beta+\alpha \frac{K_{0}(\alpha, \alpha)}{K_{1}(\alpha, \alpha)}\right) & \text { if } \quad r=j=0, \\ -\frac{\alpha^{r-1}\left(1-|\beta|^{2}\right)^{\frac{1}{2}}(\alpha-\beta)^{2}}{\sqrt{K_{1}(\alpha, \alpha) K_{r}(\alpha, \alpha) K_{r+1}(\alpha, \alpha)}} & \text { if } \quad j=0, r \geqslant 1, \\ \frac{\sqrt{K_{r}(\alpha, \alpha) K_{r+2}(\alpha, \alpha)}}{K_{r+1}(\alpha, \alpha)}, & \text { if } \quad j=r+1, \\ -\alpha\left(\frac{|\alpha-\beta|^{2}}{K_{r}(\alpha, \alpha)}-\frac{K_{r}(\alpha, \alpha)}{K_{r+1}(\alpha, \alpha)}\right), & \text { if } 1 \leqslant j=r, \\ -\frac{|\alpha-\beta|^{4} \alpha^{r-j+1}}{\sqrt{K_{r}(\alpha, \alpha) K_{r+1}(\alpha, \alpha) K_{j}(\alpha, \alpha) K_{j+1}(\alpha, \alpha)}}, & \text { if } \quad 1 \leqslant j<r, \\ 0, & \text { otherwise. }\end{cases}
$$




\section{Acknowledgements}

The work of the first author (F. Marcellán) was supported by Dirección General de Investigación, Ministerio de Educacin y Ciencia of Spain, under grant BFM 2003-06335-C03-02, and INTAS Research Network NeCCA INTAS 03-51-6637. The work of the second author (J. Hernández) was supported by Fundación Universidad Carlos III de Madrid.

\section{References}

[1] M. Buhmann, A. Iserles, On orthogonal polynomials transformed by the $Q R$ algorithm, J. Comput. Appl. Math. 43 (1992), 117-134.

[2] Ja. L. Geronimus, Polynomials orthogonal on a circle and their applications, Amer. Math. Soc. Providence RI (1962), 1-78.

[3] E. Godoy, F. Marcellán, An analogue of the Christoffel formula for polynomial modification of a measure on the unit circle, Boll. Unione Mat. Ital. Sez. A 5 (no. 1) (1991), 1-12.

[4] E. Godoy, F. Marcellán, Orthogonal polynomials and rational modifications of measures, Canad. J. Math. 45 (no. 5) (1993), 930-943.

[5] L. Golinskii, P. Nevai, and W. Van Assche, Perturbation of orthogonal polynomials on an arc of the unit circle, J. Approx. Theory 83 (1995), 392-422.

[6] U. Grenander, G. Szegö, Toeplitz Forms and their Applications, 2nd edition, Chelsea publishing Co., New York, 1984.

[7] T. Kailath, B. Porat, State-space Generators for Orthogonal Polynomials, in: Prediction Theory and Harmonic Analysis, The Pesi Masani Volume, V. Mandrekar and H. Salehi eds., Nort-Holland (1983), 131-163.

[8] F. Marcellán, J. Hernández, L. Daruis, Spectral transformations for Hermitian Toeplitz matrices, (submitted).

[9] B. Simon, Orthogonal Polynomials on the Unit Circle, Amer. Math. Soc. Providence, RI Colloquium Publ. Series 54, 2005.

Francisco Marcellán and Javier Hernández

Departamento de Matemáticas

Universidad Carlos III de Madrid

Avenida de la Universidad 30

28911 Leganés

Spain.

e-mail: pacomarc@ing.uc3m.es

jhbenite@math.uc3m.es 